\title{
Desarrollo Local Basado en Conocimiento e Innovación: Caso Agrópolis del Norte
}

\section{Local Development Based on Knowledge and Innovation: Case of study Agrópolis del Norte}

\author{
Katherine Sánchez Zambrano', Luis Alfonso Escobar Jaramillo², Myriam Sánchez Mejía3, \\ Gabriel Concha Arango 4
}

\begin{abstract}
Este artículo inicialmente aborda a la gestión del conocimiento como factor de desarrollo de una sociedad o de una región. Gestionar conocimiento implica generar condiciones para la creación e intercambio de conocimiento, para apoyar estos procesos es necesario articular sistemas organizativos que promuevan la activación de capitales intangibles y endógenos, como el conocimiento. Bajo esta concepción teórica se construye un Sistema Local de Innovación (S.L.I) para la Agrópolis del Norte, con el propósito de estimular dinámicas que integren y movilicen los actores sociales de los nueve municipios del Norte del Valle del Cauca (Colombia), que constituyen la Agrópolis del Norte, hacia un desarrollo basado en capitales financieros y no financieros (o intangibles), a través de la gestión del conocimiento.
\end{abstract}

Keywords: agrópolis del norte; desarrollo local; sistema de innovación; gestión del conocimiento; articulación de actores; institucionalidad.

This article addresses the management of knowledge as a development factor of a society or region. Knowledge management involves generating conditions for the creation and exchange of knowledge, to support these processes is necessary to articulate organizational systems that promote the activation of endogenous and intangible capitals, such as knowledge. Under this theoretical conception, a Local System of Innovation (L.S.I) is built for Agrópolis del Norte, for the purpose of stimulate integrating and mobilizing dynamics for social actors of nine municipalities in north Valle del Cauca (Colombia), which constitute the Agrópolis del Norte, towards development of financial and non-financial capital through knowledge management.

Keywords: agrópolis del norte; local development; innovation system; knowledge management; articulation of stakeholders; governance.

\footnotetext{
Universidad del Valle. Dirección: Ciudad Universitaria Meléndez, Calle I3 No 100-00 Edificio 344 Oficina 2004. Cali -Colombia. Phone: 57 (2) 3212159.E-mail: 'kszambrano@gmail.com, ${ }^{2}$ lescobar@univalle.edu.co

${ }^{3}$ Directora de Corporación Biotec (Centro de Investigación e Innovación en la agricultura, agroindustria y Bioindustria). Dirección: Km. 17 recta Cali - Palmira CIAT. Palmira (Valle) - Colombia. Phone: 57 (2) 4450 I I4 / 57 (2) 4450 I I5. E-mail: myriams@cgiar.org

${ }^{4}$ Universidad delValle. Dirección:Avenida 9N \# 5N - 36. Of. I04. Cali - Colombia. Phone: 57 (2) 374I55I. E-mail: conchagabriel@gmail.com
}

ISSN: 07 I8-2724. (http://www.jotmi.org)

Journal of Technology Management \& Innovation (c) Universidad Alberto Hurtado, Facultad de Economía y Negocios. 


\section{Introducción}

Un Sistema de Innovación "describe el conjunto de organizaciones, tanto empresariales como institucionales que dentro de un determinado ámbito (que puede ser el geográfico), interactúan entre sí con la finalidad de asignar recursos a la relación de actividades orientadas a la generación y difusión de conocimientos sobre los que se portan las innovaciones, que en últimas son la base del desarrollo económico". (Acosta et. ál., 2006: 20-2I; Buesa, 2002). Por tanto, a través de los Sistemas de Innovación se configuran nuevos escenarios de cambios tecnológicos, organizativos e institucionales tanto a nivel de una empresa, como a nivel de una sociedad (Weber, 2005).

De esta forma, en el sistema se conforma una red de organizaciones $o$ actores claves, que constituyen el origen para que las sociedades creen, almacenen y transfieran conocimiento, habilidades y artefactos, que contribuyen con la innovación. Desde esta perspectiva, el desempeño en innovación de una economía depende no sólo de cómo se comportan las organizaciones individuales aisladamente, sino también de cómo interactúan entre sí, y de su interacción con instituciones sociales como los valores, normas y marcos legales (OCDE, 2007).

Por consiguiente Freeman (1998), considera a la innovación como un proceso complejo, sistemático y acumulativo, con incertidumbre, endógeno a la empresa o territorio, con una constante evolución e incluye en su análisis los factores institucionales y organizacionales, así como los procesos de aprendizaje.

Bajo esta base teórica se afirma, que el perfeccionamiento de un territorio (o región) orientada por la eficiencia de las ventajas comparativas de su economía, a una región con una economía basada en la innovación se logra identificando y potencializando capitales intangibles, como el conocimiento, que son poco estimulados y gestionados como motor del desarrollo regional. Para lograr ese perfeccionamiento, se propone promover cambios e innovaciones estructurales, que involucran contextos sociales, productivos, institucionales y culturales, $y$ que se interrelacionan en un entorno sistémico, abierto y complejo, a través de un Sistema Local de Innovación.

De este modo, la escala geográfica reducida (es decir, un entorno local) se constituye en un factor importante para la definición de política pública, programas y proyectos orientados al desarrollo económico y social. En la actualidad, la literatura da cuenta de numerosas experiencias (ver: Cravacuore, llari y Villar, 2002) donde se han hecho apuestas por configurar y articular segmentos territoriales que poseen características especiales en: capitales, equipamientos y condiciones geográficas, que facilitan la generación de escenarios para viabilizar el desarrollo y mejorar las condiciones de vida de los habitantes de cada territorio, y en donde la gestión de conocimiento y la gobernanza colectiva juega un papel decisorio. (Fernández 20 I0; Aranguren et. ál., 20 I0).

En consecuencia, hacer apuestas regionales basadas en conocimiento e innovación, demandan estructurar una visión conjunta de región, que indudablemente se orientaría al mejoramiento de los niveles de bienestar y calidad de vida de los habitantes de un territorio. No obstante, también requiere la articulación de actores sociales e institucionales que configuren escenarios de gobernanza en el ámbito local, esto como nodo básico para edificar un Sistema de Innovación Local. Es precisamente, este proceso, el cual, se ha estructurado para una pequeña región conformada por nueve (9) municipios del Norte delValle del Cauca - Colombia (Ver Plano I), que denominamos Agrópolis del Norte, y será el objeto de estudio en este trabajo.

Puntualmente, de Agrópolis del Norte es necesario destacar en esta subregión, que todos los centros urbanos que la componen, tienen una clara vocación hortofrutícola y función agroindustrial, donde la totalidad de sus coeficientes son significativamente mayores en la unidad.

Para explicar este proceso, se han estructurado cuatro (4) apartados, siendo este el primero de introducción, un marco que aborda las bases conceptuales de la Gestión de Conocimiento en entornos locales constituye el segundo apartado, seguido de una presentación de la orientación del Sistema Local de Innovación para la Agrópolis del Norte y el cuarto y último apartado evidencia los resultados del proceso.

\section{Gestión de Conocimiento en entornos locales}

Se entiende que la iniciativa Agrópolis del Norte se constituye como una propuesta fundamentada en los principios y prácticas de la Teoría del Desarrollo Basado en Conocimiento (Carrillo, 2005), donde la organizacional local, las redes sociales, y el reconocimiento de nuevos capitales, adicionales a los financieros, son un aporte esencial para la planificación y gestión del desarrollo regional (Sánchez, 2007).

De esta forma, con el conocimiento como factor movilizador de los capitales se configuran nuevos paradigmas de intervención para la promoción del desarrollo regional, donde los elementos claves son: la interconexión y la interactividad, la acción colectiva, la escala geográfica reducida, la escala virtual amplia, el constructivismo, la intersubjetividad, el conocimiento y las innovaciones. A su vez, estos elementos necesitan como factor promotor la confianza y creación de varias formas de capital colectivo (Boisier, 200I). 
En este marco la escala geográfica reducida, a la cual hace referencia Boisier (200I), indica la importancia estratégica de lo local para la movilización de las formas que toma el capital privado y colectivo. Como lo indica Borja y Castells (1997), la escala local se constituye en un centro de gestión de lo global en el nuevo sistema o paradigma económico, donde se aprecian tres (3) ámbitos principales: la productividad y competitividad económica, la integración sociocultural y la gestión de políticas públicas.

De acuerdo a Honrubia (2004) el diseño de políticas no es el fin último de la promoción de un desarrollo local, si bien la política pública constituye una herramienta importante para la promoción de muchas estrategias de desarrollo, también lo es el fortalecimiento de la base social y sus instituciones. Este fortalecimiento puede traducirse en el empoderamiento de la sociedad lo cual, sí es herramienta fundamental para un proceso de largo plazo.
Es importante anotar que la formulación de la política pública no implica persé que necesariamente se cumpla, no obstante, la sociedad empoderada y sus instituciones pueden generar la movilización y dinámica que permita impulsar y concretar acciones orientadas al desarrollo local. Ahora, sí estas acciones son propuestas conjuntas de región los objetivos serán más factibles de alcanzar en la medida que la gobernanza que pueda construirse sea consolidada.

De este modo, como lo afirma Cuervo (1998) "el enfoque desarrollo local es una de las respuestas a los problemas del desempleo y desorganización económica causados por la decadencia industrial y las deslocalizaciones. Después del fracaso relativo de los proyectos organizados y aplicados por organismos públicos nacionales, la idea de utilizar procedimientos locales ha ido ganando vigencia”.

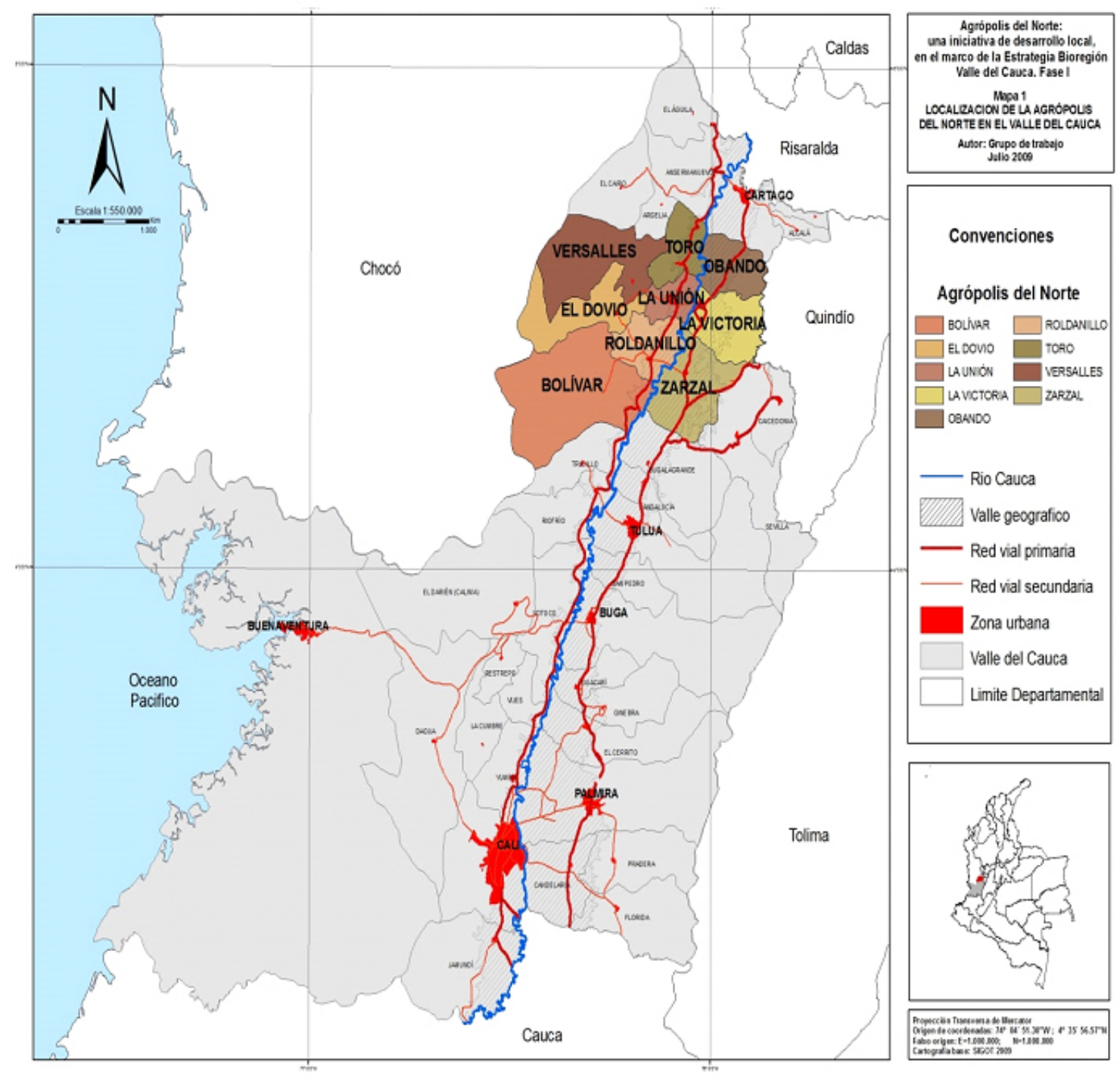

Plano I: Municipios de la Agrópolis del Norte. Fuente: Grupo Técnico Agrópolis del Norte http://agropolisdelnorte.info

ISSN: 07 I 8-2724. (http://www.jotmi.org)

Journal of Technology Management \& Innovation (C) Universidad Alberto Hurtado, Facultad de Economía y Negocios. 
Bajo esta concepción, en Europa en la década del 70 impulso políticas orientadas a fortalecer el Desarrollo Económico Local bajo agencias denominadas "Grupo de Acción Local (GAL)". Para el caso de América Latina, a finales de la década de 1990 (especialmente en Argentina y Chile) surgieron Agencias de Desarrollo y Consorcio Municipales, como respuesta a la gestión estructural de los problemas económico-sociales que en ese momento presentaban las regiones.

Vásquez-Barquero (1993 y 1997) indica que a partir del Desarrollo Local y la movilización que se genera, se produce una reestructuración del Estado y se impulsan nuevas formas en la gestión pública.A su vez, que concibe al desarrollo económico local como un proceso de crecimiento y cambio estructural que produce como resultado la movilización de capitales, aprovechamiento del entorno e introducción de innovaciones que generan el aumento de bienestar de la población de una determinada región.

Por tanto, el desarrollo local se puede interpretar como una reestructuración del estado con una nueva configuración de instituciones que promueven la activación de los recursos propios (endógenos), fortaleciendo las relaciones horizontales con redes cooperativas de actores estales y no estales, a partir del reconocimiento de las potencialidades del territorio, sus recursos y capitales financieros y no financieros.

En consecuencia, siguiendo a Honrubia (2004) el enfoque del desarrollo desde una escala local aborda una nueva perspectiva, integral y holística, combinando la planificación física (territorial), económica, social y hasta medioambiental, incorporando el criterio abajo-arriba (bottom-up por su definición anglosajona), implicando nuevos actores y niveles de actuación para la gobernanza de los procesos de desarrollo regional.

A este nivel el territorio organizado constituye la base económica domestica donde se construye la ventaja competitiva, para lo cual deben proveer un entorno igualmente competitivo; dotado de infraestructura y servicios públicos adecuados, con oferta educativa de acuerdo a la demanda del sistema productivo, apoyados en centros de investigación y desarrollo entre otros procesos (Millán 1994). En este sentido el conocimiento se convierte en un factor movilizador del desarrollo, constituyendo un entorno donde es posible la innovación de las estructuras productivas y el sistema de capitales locales, y por ende la competitividad de las regiones. Por ello, es necesario un eficaz vínculo entre el mundo educativo y el productivo. (Alvarado-Borrego, 2009), de este modo, se hace palpable la relación entre conocimiento, competitividad, sociedad y desarrollo, que convergen a procesos de Gestión del Conocimiento.
Arbonies (2007) indica que la gestión del conocimiento es la concatenación de proyectos y estrategias que conduce a la generación, transferencia y trasformación del conocimiento. Sin embargo, hay dos (2) aspectos relacionados con el conocimiento que se deben tener en cuenta en la promoción del desarrollo regional:

I. El conocimiento no se puede gestionar en el sentido clásico de la palabra. Lo que se puede gestionar es el proceso en el que se intercambia y se crea conocimiento.

II. “El conocimiento es un acto complejo, no puede ser planificado, ni demandado a voluntad. Es emergente y surge en determinadas condiciones, mientras que no lo hacen otras, sin que de momento podamos controlar y manipular el infinito abanico de interacciones y condiciones que lo promuevan" (Arbonies, 2007:21).

En este sentido, esta perspectiva proporciona los elementos conceptuales que permitirían contextualizar la construcción social de organizaciones locales orientadas a gestionar el desarrollo regional basado en la gestión del conocimiento como motor de la prosperidad colectiva. De esta forma, se espera que las organizaciones sociales sean organismos vivos, que reflejen sistemas adaptativos complejos con agentes locales que actúan de forma emergente e impredecible en su aporte a un objetivo compartido de desarrollo (Stancey 200I). Como corolario de estos argumentos Arbonies (2007) indica que "para gestionar el conocimiento, se deben articular sistemas organizativos que aprovechan las capacidades y el conocimiento de todas las personas frente a la vieja organización que lo despilfarra de modo poco inteligente".

Con esta referencia conceptual es posible comprender que el Proyecto "Agrópolis del Norte: una iniciativa colectiva de desarrollo local, basada en conocimiento en el marco de la Estrategia Bioregión Valle del Cauca. Fase l" está orientado a promover la articulación de actores sociales alrededor de un Sistema Local de Innovación (SLI), con el propósito de estimular dinámicas que integren y movilicen los actores sociales de los nueve municipios de la Agrópolis del Norte, hacia un desarrollo basado en su sistema de capitales (financieros y no financieros o intangibles), a través de la gestión del conocimiento.

\section{Hacia un Sistema Local de Innovación en la Agrópolis del Norte}

El pensamiento vanguardista propuesto por el Desarrollo Basado en Conocimiento (DBC) no sólo se enfoca en el flujo de conocimiento entre agentes, sino que en profundidad se encamina a los sistemas de valor basados en el conocimiento. Entendido que estos sistemas son 
colectivos humanos que persiguen deliberadamente un desarrollo integral sostenible con particular énfasis en los bienes intangibles o de conocimiento. (Carrillo 2004: 5)

Siguiendo esta misma concepción, Boisier (2002) indica que el DBC se constituye como una estrategia integral de desarrollo fundamentada en identificar, sistematizar y desarrollar el universo de capitales intangibles por lo cual, el DBC constituye un proceso y estructuras sistémicas, abiertas y complejas.

Siguiendo a Carrillo y Boisier se puede argumentar que el conocimiento es un activo predominante dentro de las dinámicas de crecimiento y desarrollo para una región (ver figura I). Por ello, el conocimiento es un factor generador de valor, que envuelve procesos de aprendizaje y de innovación.

En este sentido, Ludvall (2005) expone tres (3) aspectos interesantes:

I. El conocimiento puede aparecer en el proceso productivo como un factor (cualificación o inversión en $I+D$ ) y como un producto (patente o innovaciones).
II. El conocimiento bajo determinadas circunstancias, puede ser de propiedad privada, y/o comprado y/o vendido en el mercado como una mercancía.

III. La economía del conocimiento trata en gran medida de especificar las condiciones para que el conocimiento se genere como una mercancía "normal" en la línea de los productos tangibles reproducibles.

El conocimiento involucrado en procesos productivos trae inmerso implicaciones de reconversión productiva, fortalecimiento de sistemas locales de ciencia y tecnología, focalización de ayuda financiera y técnica, promoción de ventajas competitivas, así como repercusiones sociales y políticas en todos estos procesos (Boisier 2002). De este modo, el reconocimiento y la inclusión del factor conocimiento en todos los procesos productivos, sociales, políticos, e incluso culturales de la región, originan un proceso de cambio estructural que se orienta a una transformación sistémica del propio territorio. Buscando este proceso de transformación en la Agrópolis del Norte, se promueve la construcción de un SLI (ver figura I).

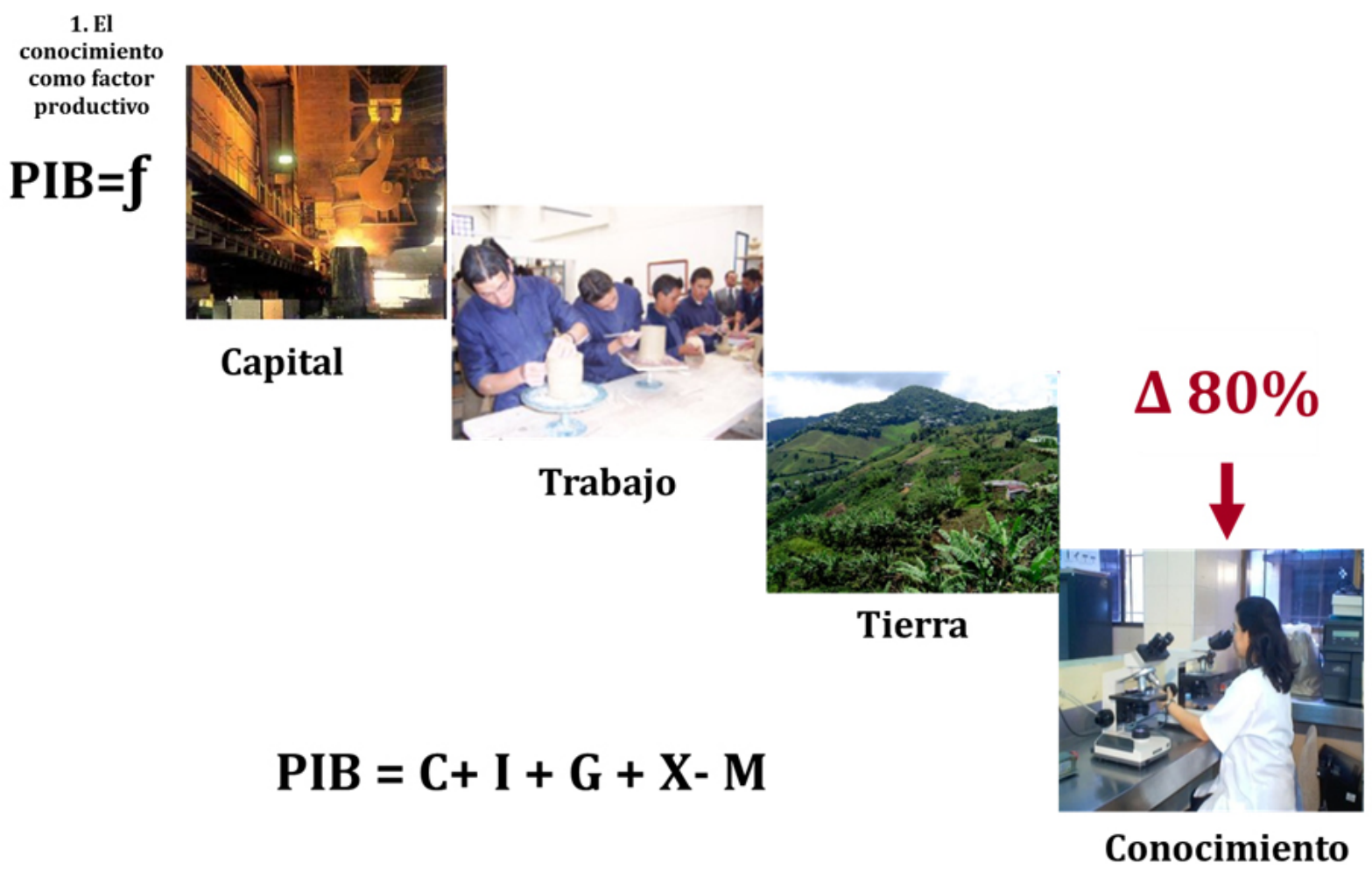

TOMADO DE LA PRESENTACIÓN DE LA LEY 1286 DE 2009, DR. JUAN FRANCISCO MIRANDA DIRECTOR DE COLCIENCIAS

Figura I: Conocimiento y Crecimiento Económico. Fuente: Presentación de la Ley I286, Dr. Juan Francisco Miranda, Director de Colciencias.

ISSN: 07 I8-2724. (http://www.jotmi.org)

Journal of Technology Management \& Innovation (c) Universidad Alberto Hurtado, Facultad de Economía y Negocios. 
Según Bennet A y D (2007) los S.L.I son entendidos como el conjunto de agentes y procesos que permiten la competitividad y el bienestar de las comunidades, empoderadas por la movilización de capitales, especialmente la movilización del (capital) conocimiento.

Estos S.L.I se constituyen por una red de organizaciones, empresas e individuos orientados a dar un uso social y económico a nuevos productos, nuevos procesos y nuevas formas de organización, conjuntamente con las instituciones y políticas que afectan su comportamiento y desempeño.

El concepto de sistemas de innovación no solamente incluye a los oferentes de la ciencia sino también a la totalidad de actores y sus interacciones, involucrados en la innovación. Se extiende más allá de la creación de conocimiento para incluir los factores que afectan la demanda por el uso de conocimiento en formas novedosas y útiles. (Banco Mundial, 2008: 16).

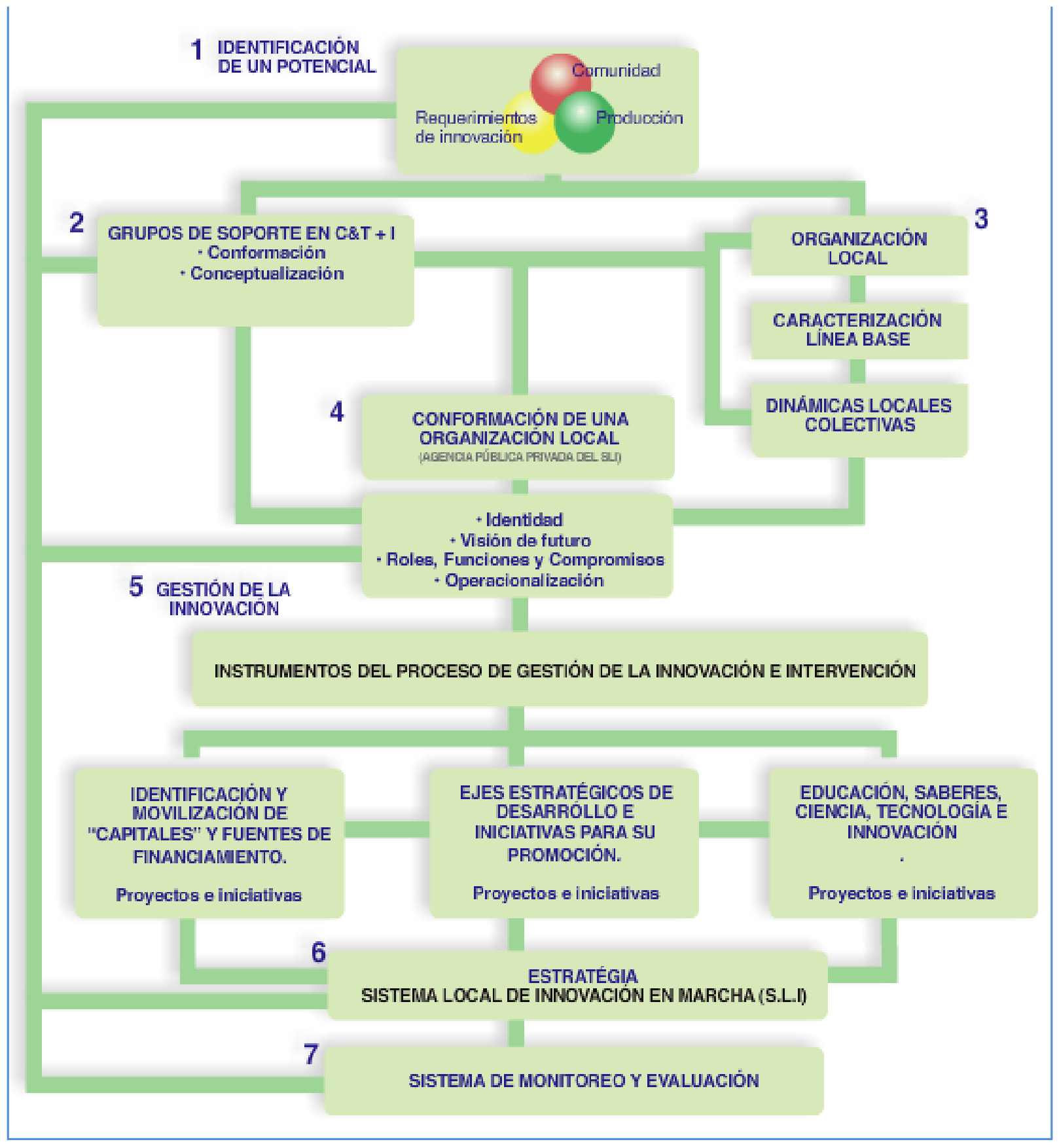

Figura 2: Hacia un Sistema Local de Innovación en la Agrópolis del Norte. Fuente: Sanchez (2009)

ISSN: 07 I8-2724. (http://www.jotmi.org)

Journal of Technology Management \& Innovation (c) Universidad Alberto Hurtado, Facultad de Economía y Negocios. 
De este modo es que se entiende la importancia de los procesos de innovación, dada la relación y sinergia entre conocimiento, tecnología y productividad que son aspectos que anteceden y determinan el éxito de la innovación, es decir, la innovación es un resultado de la activación del conocimiento (Arboníes, 2006). En este orden de ideas Rotwell (2004) indica que la innovación es un proceso de personas ("Innovation is a people process"), tal como lo indica la figura 3.

Partiendo de que las innovaciones dependen del conocimiento y de las personas, los procesos y requerimientos de innovación son propios del sistema. Cuando se habla de innovación no solo se plantea una innovación productiva, sino innovaciones sociales que se relacionan con cambios o trasformaciones de estructuras y formas de organización de los diversos actores. Adicionalmente, estas innovaciones sociales se interrelacionan con esas necesidades del sector productivo o gubernamental para acceder e intervenir el territorio.

La base de los procesos se encuentra en el conocimiento, no obstante, la materia prima para la generación de conocimiento es la información, dado que permite al sistema comprender y describir su entorno o bien, describirse a sí mismo (Luhmann, 1997). En consecuencia, "conocer supone información, pero, comprender supone conocimiento" (Boisier, 200I). La comprensión y procesamiento de información involucra en muchas ocasiones procesos de aprendizaje y capacitación. Por ello, la utilización de la información requiere (aunque no en todos los casos) herramientas y elementos que se adquieren sólo con el aprendizaje (o capacitación). Lo importante y ventaja de estos tiempos, es que la transferencia y difusión, e incluso almacenamiento de información cuenta con Tecnologías de la Información y Comunicación para que toda la sociedad (o actores de un entorno) pueda acceder a ella.

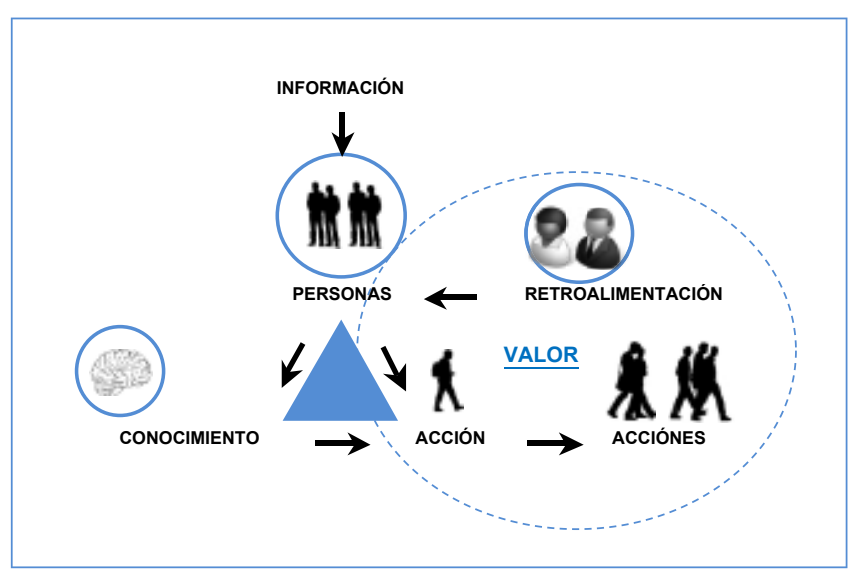

Figura 3. Movilización de Conocimiento. Fuente: Bennet A y D (2007).
Lo anteriormente descrito comprende la base teórica y conceptual en el cual se promueve la Agrópolis del Norte como un SLI (ver figura 4). De lo expuesto, los aspectos que se destacan para el caso de estudio son:

- La escala geográfica reducida. Donde lo local desempeña un papel fundamental porque se considera como el espacio más adecuado para articular y gestar asociaciones y acciones. La cooperación entre actores es más factible, en la mayoría de casos los actores en una escala local tienen conocimiento previo y relaciones ya establecidas entre ellos, además estos actores son más consientes y conocedores de las fortalezas y necesidades de su territorio (o entorno cercano). Estos procesos se robustecen si se posee un visón conjunta, es decir, se define un norte para el cual se direccionan todas las estrategias y acciones.

- El conocimiento es el generador de valor por excelencia. Teniendo esta premisa es necesario "Gestionar Conocimiento", como factor clave para la movilización de acciones encaminadas al mejoramiento y reestructuración continua del territorio, de la sociedad, de las instituciones, del sector productivo, de todo el sistema.

- La información es un insumo necesario para conocer y analizar el entorno. Por ello, se hace conveniente facilitar el acceso a la información y a los datos que caracterizan ese entorno.

- Los procesos de aprendizaje y capacitación debe estar acorde a las necesidades y fortalezas del territorio. Entonces, debe existir un vínculo entre el mundo educativo, el mundo productivo y las necesidades del entorno.

- La descripción de un SLI permite hacer visible las relaciones complejas que se presentan en un entramado de interacciones entre actores $y$ las necesidades $y$ fortalezas (demandas y ofertas) del territorio para los procesos de activación de conocimiento y de innovación.

En el contexto de la Agrópolis del Norte, el marco de referencia conceptual de los SLI evidencia un gran encadenamiento de ideas, que a partir del conocimiento como factor fundamental de movilización del sistema de capitales, incorpora en todos los procesos la complejidad de las relaciones que se generan en un sistema. La transversalidad del factor conocimiento hace que surjan ideas y propuestas de desarrollo complejas. En este sentido, cuando se plantean aspectos como estos, se retoma a Luhmann cuando dice: "no se puede ganar un juego complejo con jugadores y estrategias simples".

De manera puntual, el Proyecto "Agrópolis del Norte: una iniciativa colectiva de desarrollo local, basada en cono-

ISSN: 07I 8-2724. (http://www.jotmi.org)

Journal of Technology Management \& Innovation (c) Universidad Alberto Hurtado, Facultad de Economía y Negocios. 
cimiento en el marco de la Estrategia Bioregión Valle del Cauca. Fase I" promueve tres aspectos que se interrelacionan cuando su propósito es movilizar el conocimiento en un SLI:

I. Formular e implementar estrategias para la articulación de actores sociales (locales e internacionales) alrededor de la iniciativa de desarrollo local de la Agrópolis del Norte.
II. Establecer un sistema de información para la toma de decisiones de la Agrópolis del Norte.

III. Diseñar un proyecto pedagógico de aprendizaje-acción para la formación de actores sociales, en nuevos esquemas de la cadena de valor de la investigación, para fortalecer la relación Universidad-Estado-Empresa.

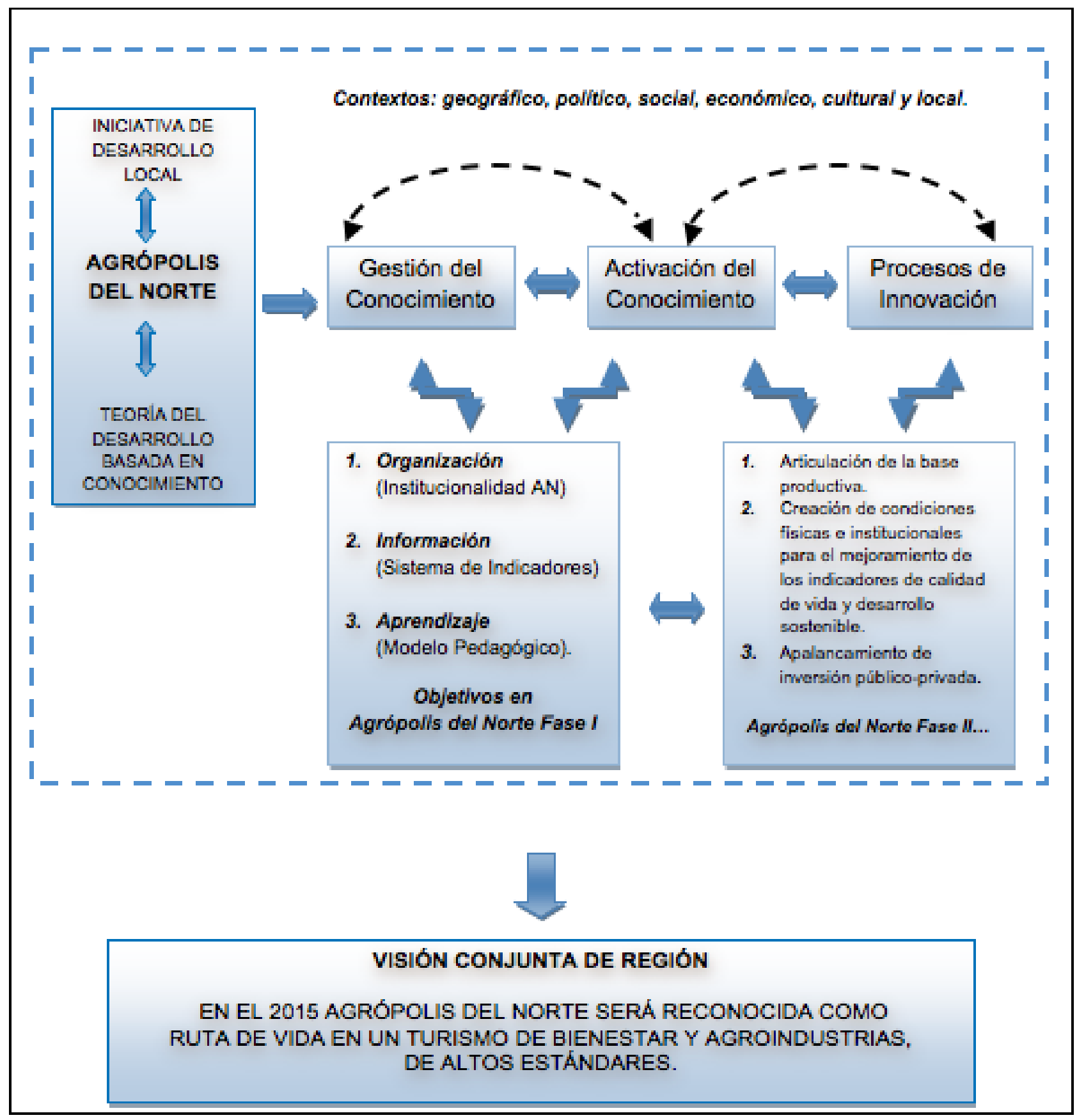

Figura 4:Agrópolis del Norte como Propuesta de Desarrollo Local y Colectivo Basada en Conocimiento $<\cdots>$ Procesos Continuos de Retroalimentación. Fuente: Sanchez K (20I I). 
El primer objetivo de articulación y movilización de actores se efectúa para construir consenso alrededor de una identidad de región (reconociendo los factores económicos, sociales y ambientales que hacen potencialmente atractiva la intervención) y una visión de futuro de la Agrópolis del Norte. En los sistemas y más aún cuando son locales se hacen, efectivos, reales y frecuentes los procesos de interacción entre sus habitantes, pero, lo que realmente cohesiona a los actores es una identidad colectiva reconocida (Arocena 1995).

En el segundo objetivo de constituir un Sistema de Información, constituye una herramienta fundamental para el sector productivo y gubernamental al forjarse como un instrumento para la toma de decisiones e intervenciones en el territorio. Por ello, los Sistemas de información se conciben como una forma de ganar y generar acceso al conocimiento (Boisier 200I). En el anexo I puede observar los principales desarrollo del sistema de información que se han gestado en torno a la ejecución del Proyecto, y que son soporte para la puesta en marcha del SLI Agrópolis del Norte.

En tercer y último objetivo que se direcciona a los aspectos educativos y pedagógicos, se busca de un marco estratégico para la educación regional que de respuesta a las necesidades del entorno y su demanda productiva. Por ello, Alvarado-Borrego (2009) infiere que se requiere un eficaz vínculo entre el mundo educativo y el productivo, en esta nueva sociedad, la educación se convierte en un instrumento imprescindible para sustentar el cambio y reproducirlo. Los sistemas educativos deben movilizarse hacia la sociedad del conocimiento y generar el conocimiento significativo que se requiere. En el anexo 2 se detallan los elementos que permiten dar a entender la puesta en marcha de la educación como factor de movilización del conocimiento en la Agrópolis del Norte. Con estos elementos, se puede argumentar con Arboníes (2007), que Agrópolis del Norte apunta hacia cambios educativos que promueven una nueva pedagogía, nuevos formatos y contenidos curriculares, apuestas para pasar de la enseñanza al aprendizaje, y acercarse a las necesidades de la empresa, con iniciativas como la TecnoAcademias, entre otros ejercicios conceptuales derivados de este Proyecto de Investigación.

Como corolario de todos estos argumentos se deduce que en la Agrópolis del Norte se está gestado lo que Arboníes (2007) denomina un cuadro sistémico no fragmentado, al establecerse en:

PERSONAS y enfocarse en su capacitación, la cantidad de información con la que cuentan, su competencia y su participación como factor indispensable en proyectos.
ORGANIZACIÓN como espacios y rutinas creativas que permite la creación de conocimiento, innovación y aprendizaje.

TERRITORIO INNOVADOR como un sistema de innovación donde es posible la asociación en redes y clúster, la colaboración público privado para el desarrollo de la región.

En este sentido se deduce que la articulación de actores, los modelos pedagógicos que movilizan el conocimiento aplicable al sistema de capitales, y la información para la caracterización de los sistemas productivos, demanda la comprensión de las potencialidades territoriales de una región que se concibe como un SLI. De esta forma, en la siguiente sección se presentan las condiciones territoriales que hacen de la Agrópolis del Norte una región potencialmente promisoria para el desarrollo a partir de la gestión del conocimiento. En ese contexto, la siguiente sección se centraría en mostrar el proceso de articulación de actores en el territorio estudiado, indicando que están listas las condiciones institucionales y que el proceso de investigación muto a una iniciativa de desarrollo local en marcha.

\section{Resultados}

La articulación de actores sociales en un SLI es un proceso en el que se generan sinergias capaces de consensuar y marcar la dirección que se debe seguir un territorio que pretende basar su desarrollo en la gestión del conocimiento. Esta articulación de intereses convergentes, en lo privado y lo público, permite la configuración de un conjunto de estrategias de desarrollo local orientadas a la movilización de los diversos sistemas de capitales en una región. En este orden de ideas, la articulación de actores sociales en el territorio, demanda la formación, desarrollo y asociación de instituciones empoderadas socialmente que gobiernen las estrategias de desarrollo regional en un SLI.

Arbonies (2007) argumenta que en todos estos procesos no sólo se trata de liderato, de tener objetivos comunes, y de dar poder a las personas, más bien se trata de un sistema vivo, que permite crear un organismo en donde se generan metas comunes, donde las expectativas de unos y otros se retroalimentan continuamente, donde existe libertad y poder de decisión con ciertas dosis de sentido y propósito común.

En esta misma línea Arbonies argumenta que "los sistemas generalmente presentan un flujo general de decisiones, pero esto constituye un pálido reflejo de las innumerables interacciones entre gentes que intercambian y crean conocimiento para llegar a la innovación". Por ello, el SLI de la Agrópolis del Norte (ver figura 5) esboza las relaciones que deben generarse dentro del territorio para activar el conocimiento como factor de producción y generación de procesos de

ISSN: 07 I8-2724. (http://www.jotmi.org)

Journal of Technology Management \& Innovation (c) Universidad Alberto Hurtado, Facultad de Economía y Negocios. 
innovación regional a partir de los desafíos que se han marcado en la caracterización territorial (ver sección 4).

De acuerdo a Cravacuore et. al (2000) el papel de los actores (o diversos agentes) en los procesos de innovación en el ámbito local se puede definir en tres postulados:

I. El proceso innovador se centra en los actores estatales y su poder de cambio, y considera que existen actores políticos o burocráticos que proponen el cambio organizacional.

II. La innovación encuentra su énfasis en el entorno de los actores y el conjunto de retos y alteraciones que los cambios sociales y tecnológicos provocan. Esta perspectiva indica que el cambio es originado por presiones de los actores públicos no estatales o privados.

III. La innovación encuentra su origen en la existencia de una trayectoria histórica generadora de una cultura organizacional innovadora y de valores colectivos que estimulan el desarrollo de la innovación de sus miembros.
Se colige de lo anterior, que es necesaria la organización del territorio desde sus actores e instituciones para promover un DBC. En el caso de Agrópolis del Norte la identificación de actores sociales y su movilización, ha implicado una serie de requerimientos de información y formación de capacidades para la formulación de políticas públicas y estratégias de intevención. De esta forma, el proceso de articulación y movilización de actores sociales en esta región se ajusta a la configuración de escenarios para lo que Boisier (2002) denomina el nuevo entorno del desarrollo regional, al referirse a tres momentos que pueden perimir el posicionamiento de una sociedad del conocimiento: el escenario contextual o apertura, el escenario estratégico que da origen a nuevas formas de organización, y el escenario político que invoca la reingeniería de la gestión territorial para promover el desarrollo regional.

La fingura 5 indica el proceso de articulación de actores en la Agrópolis del Norte, en el que se describen tres etapas que constituyen la metodología y estrategia seguida para la puesta en marcha de la iniciativa regional Agrópolis del Norte.

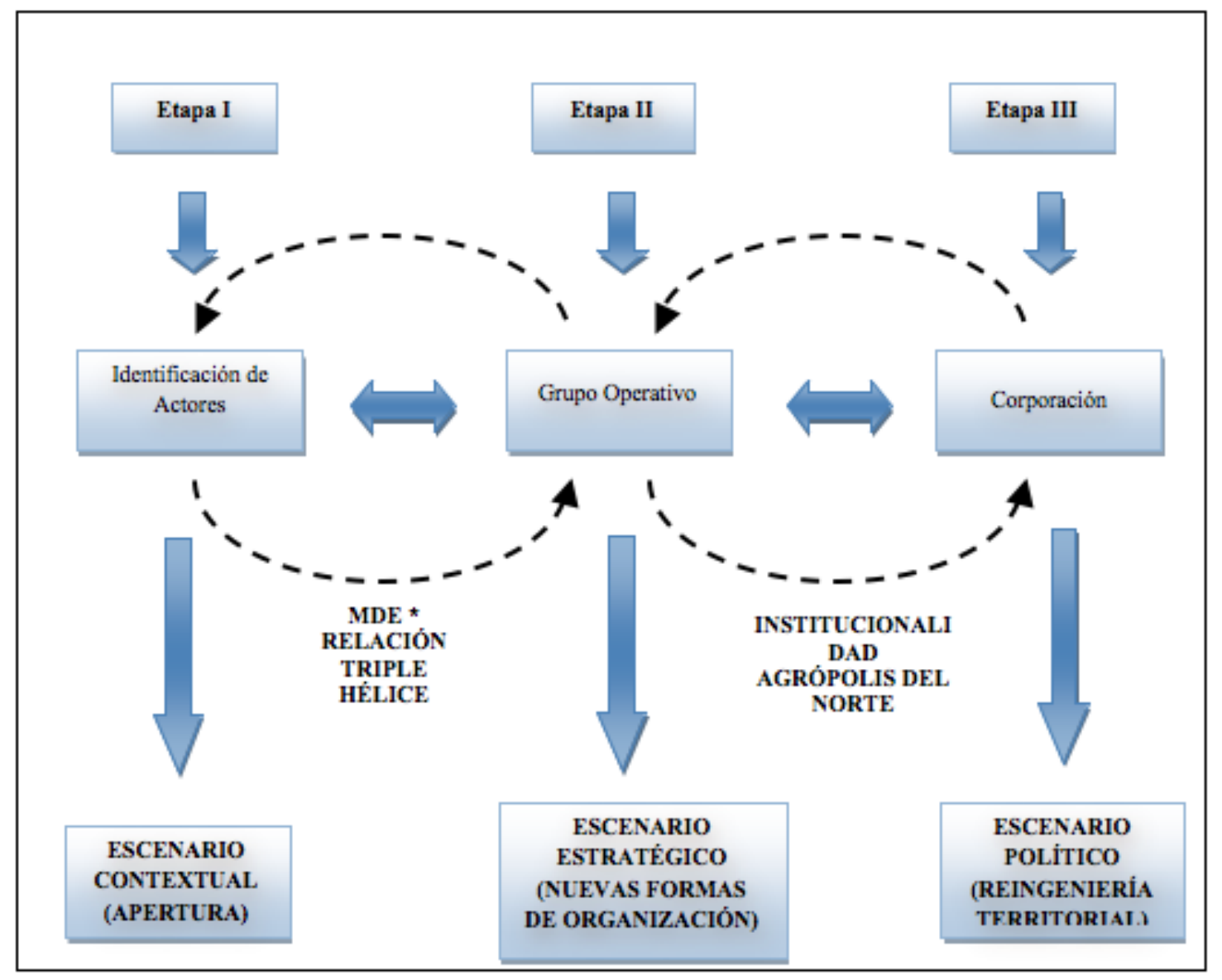

Figura 5 proceso de articulación de actores en la agrópolis del norte.

* MDE: Memorando d de Entendimiento. Documento que formaliza el respaldo de las instituciones a la iniciativa Agrópolis del Norte $<\cdots>$ Procesos Continuos de Retroalimentación. Fuente: Sanchez K (20II).

ISSN: 07 I8-2724. (http://www.jotmi.org) 


\section{Etapa I (Apertura)}

Se reconoce, vincula y capacita (contextualización) a los actores. En principio se trabajó con la sociedad de base, pasando por organizaciones (sociales y empresariales) hasta las alcaldías. De esta convocatoria surgió la iniciativa de comprometer las alcaldías, (esta propuesta surge desde la misma comunidad de base) y en respuesta a ello se efectúa el 22 de febrero del año 2010 la Firma del Memorando de Entendimiento (MDE), este documento de carácter simbólico indica el acompañamiento y respaldo de las alcaldías con la iniciativa Agrópolis del Norte.

\section{Etapa II (Nueva forma de organización)}

Una vez las alcaldías firman el MDE se propone constituir un grupo de trabajo, que se reunirá periódicamente, El Grupo Operativo se conformo por Coordinadores de UMATAS y Secretarios de Planeación, se contó con la participación de mínimo dos (2) personas por municipio (representantes de las alcaldías). En el proceso de trabajo con este grupo se adhirieron actores empresariales y sociales, como Grupo C. Lozano, Comfandi y Fundación Enlace. En este sentido se constituyó un grupo interdisciplinario que integra los cuatro (4) municipios (Zarzal, Roldanillo, La Unión y La Victoria).

Este Grupo Operativo, constituyo una fuente de divulgación y promoción para la iniciativa Agrópolis del Norte, además de otros logros. Del trabajo del Grupo Operativo se destacan tres (3) aspectos: I) establecimiento de una visión conjunta de región. II) promoción de la estructuración de un Plan Estratégico Educacional para la Agrópolis del Norte. III) Promoción del proyecto Tecnoacademia Agrópolis del Norte.

\section{Etapa III (Institucionalidad)}

Es la configuración de la "Corporación para el Desarrollo del Sistema Local de Innovación Agrópolis del Norte", para dicha corporación a la fecha se han elaborado los Estatutos y Acta de Inicio. Esta Corporación es la encargada de continuar con los procesos que se han generado desde la primera fase del proyecto Agrópolis del Norte. Asu vez esta Corporación posee una Grupo Técnico Asesor conformado por La Universidad del Valle, Corporación Biotec y la Universidad San Buenaventura.

La CORPORACIÓN SLI AGROPOLIS DEL NORTE tiene como misión fundamental, promover y desarrollar las acciones, decisiones, investigaciones, innovaciones, inversiones y procesos empresariales y sociales requeridos para incrementar la competitividad y el desarrollo local colectivo, integrado, coordinado y complementario, de los nueve (9) municipios del Norte del Valle del Cauca: Roldanillo, Zarzal,
La Unión, La Victoria, Bolívar, El Dovio, Toro, Obando y Versalles, que se reconocen como la Agrópolis del Norte. Este desarrollo estará basado en el reconocimiento de múltiples "capitales" de las comunidades locales, teniendo en cuenta saberes, prácticas, recursos naturales empresariales y sociales, y su movilización, especialmente la movilización del capital conocimiento, como fundamento estratégico para dinamizar las potencialidades y complementariedades mediante las cuales se generen las dinámicas necesarias para la construcción social de un Sistema Local de Innovación. 


\section{Referencias}

ACOSTA, P. (2009). Ciudades de América Latina en La Sociedad del Conocimiento: Experiencias de Investigación, Innovación y Creatividad. División de Planeación Estratégica y Evaluación de Colciencias. Bogotá, Colombia.

ALVARADO-Borrego, A. (2009). Vinculación Universidad Empresa y su Contribución al Desarrollo Regional. Revista Científica Ra Ximhai, 5 (3), 407 - 4I7. Septiembre-Diciembre de 2009.Pág. 407-4I4.

ARANGUREN, J., Larrea, M., Y Wilson, J. (20I0). "Presentación. Gobernanza y Competitividad Territorial". Ekonomiaz: Revista Vasca de Economía. 74 (2), 7 - 15.

ARBONIES, A. (2007). Conocimiento para innovar: cómo evitar la miopía en la gestión de conocimiento. 2da Edición. Díaz de los Santos S.A. Madrid

AROCENA, J. (1995). El Desarrollo Local: Un Desafío Contemporáneo. I ra Edición. Nueva Sociedad. Caracas, Venezuela.

BENNET A., Bennet D. (2007). Knowledge mobilization in the social sciences an humanities. Moving from research to action. MQI Press, USA 2007.

BOISIER, B. (200I). Sociedad del conocimiento, conocimiento social y gestión territorial. Documentos OCDE.

BOISIER, B.2002. Actores, asociatividad y desarrollo territorial en la sociedad del conocimiento. Documento de Trabajo. Informe para Cresce Minas. Um Projeto Brasileiro, Federaçao das Industrias do Estado de Minas Gerais en Belo Horizonte, Brasil.

BORJA, J., Y Castells. M. (1997). Local y global. La gestión de las ciudades en la era de la información. Editorial Taurus. España.

BUESA, M., Martínez, M., Heijs, J., And Baumert, T. (2002). Los Sistemas Regionales de Innovación en España. Una tipología basada en indicadores económicos e institucionales. Revista Economía Industrial. 347 (I), 15-32.

CARRILLO, J. (2004). Desarrollo Basado en Conocimiento. Revista Transferencia. 65 (I), 24-26.

CARRILLO, J. (2005). “¿Qué es la Economía del Conocimiento?". Revista Transferencia. 69 (I), 2-5. Cravacuore, D. (2000) La articulación de actores para el desarrollo local", en Desarrollo Local. Una revisión crítica del debate. Universidad Nacional de Quilmes y Universidad Nacional General Sarmiento, Espacio Editorial.Argentina.
CRAVACUORE, D., llari, S., Y Villar, A. (2002); La articulación en la gestión municipal. Actores y políticas. Editorial de la Universidad Nacional de Quilmes. Argentina, Buenos Aires.

CUERVO, L. (1998). Desarrollo económico local: leyendas y realidades. Revista de Estudios Regionales y Urbanos I (I), 9-24.

FERNÁNDEZ DE LUCIO, J. (20I0).Valores no epistémicos en la ciencia reguladora y en las políticas públicas de ciencia e innovación. Revista Argumentos de Razón Técnica. I3 (I), $4 \mid-56$.

FREEMAN, C. (1998). "La economía del cambio tecnológico". En Ralph L., Christopher F. (Eds), Economía de la innovación: las visiones de Ralph Landau y Christopher Freeman pp. 49-II5.

HONRUBIA, J. (2004). Globalización y desarrollo local: una perspectiva Valenciana. Hyperlink "http://dialnet.unirioja.es/ servlet/editor?codigo=603" Universitat de València. Valencia, España.

LUDVALL, B. (1999). La Base del Conocimiento y su Producción. Ekonomiaz: Revista Vasca de Economía. 45 (I), I4-37.

LUHMANN N. (1997). Sociedad y sistema: la ambición de la teoría. PAIDOS Barcelona, España.

MILLÁN C. (1994). Competitividad Internacional de Las Regiones. Cámara De Comercio de Cali, FDI, Santiago De Cali, Colombia.

OCDE. (2007) Estudios de la OECD sobre políticas de innovación. Edición en español publicada por Ministerio de Economía, Fomento y Reconstrucción de Chile.

SÁNCHEZ K. (20II). La Articulación de Actores en un Sistema Local de Innovación. Caso Agrópolis del Norte. Trabajo de Grado (en Economía). Universidad del Valle. Facultad de Ciencias Sociales y Económicas. Departamento de Economía. Colombia.

SÁNCHEZ M. (2009). Sistema Local de Innovación para la Agrópolis del Norte. Documentos de trabajo Corporación Biotec. CIAT, Palmira-Colombia.

SÁNCHEZ M. 2007. Análisis del retorno a la inversión en la cooperación científico-tecnológica. Una herramienta para la relación ciencia-sociedad en Colombia, En Acción social (Eds), Perspectivas de la cooperación internacional para el desarrollo en Colombia. Bogotá-Colombia. PP 167 - 185.

ISSN: 07 I8-2724. (http://www.jotmi.org)

Journal of Technology Management \& Innovation (c) Universidad Alberto Hurtado, Facultad de Economía y Negocios. 
STANCEY, R. (200I) Complex Responsive Processes in Organizations: Learning and Knowledge Creation. Routledge. London.

VÁSQUEZ-Barquero A. (1997). ¿Crecimiento endógeno o desarrollo endógeno?. Cuadernos del CLAEH. 78 (I) - 79 (I), III-128.

WEBER, M. (2005). Towards Environmental Innovation Systems. Springer.Alemania.

\section{NOTAS}

Articulo fundamentado en el trabajo grado de Sánchez K.: La Articulación de Actores en un Sistema Local de Innovación. Caso Agrópolis del Norte. Dicho trabajo, se efectúo en el marco del proyecto de investigación: Agrópolis del Norte: Una iniciativa de Desarrollo Local en el Marco de la Estrategia Bioregión. Fase I, en cual fue financiado por Colciencias y ejecutada por la Universidad del Valle, La Corporación Biotec y la Universidad de San Buenaventura seccional Cali.

Desarrollo Regional Agropolitano, es una idea promovida por Jhon Friedmann desde la década de 1970, el cual propone una búsqueda del desarrollo alternativo basada en actores, recursos y capacidades locales. (Helmsing, 1999). Desde la perspectiva de la estructura territorial, la imagen de lo Agropolitano se relaciona a un territorio en el que se produce "la fusión entre lo rural y lo urbano" y donde el predominio socioeconómico de lo urbano se constituye sobre una base física rural. Por lo cual, las regiones agropolitanas necesitan ir más allá, ser innovadora e imaginativas buscando en la fusión y la complementariedad entre lo rural y lo urbano. (Izquierdo, 2008).

Agrópolis del Norte es una subregión (que también se podría denominar microregión porque tiene su escala pequeña) con nueve (9) municipios: Zarzal, Roldanillo, La Unión, La Victoria, Bolívar, Versalles, El Dovio, Toro y Obando, la cual, se consolida como una unidad territorial que hoy se denomina Agrópolis del Norte la cual posee una extensión $2.493 \mathrm{~km} 2$, el II \% del territorio departamento del Valle del Cauca (Colombia). Los capitales intangibles los constituyen: capital relacional, capital intelectual, capital humano y capital estructural... Entiéndase por innovación a la implementación tecnológica de nuevos productos y procesos y/o a mejoras significativas en estos, ya sea como resultado de la difusión de conocimientos tecnológicos para lograr productos o procesos productivos con especificaciones o propiedades técnicas diferenciadas con respecto a los existentes en el mercado. (Colciencias, HYPERLINK "http://www.colciencias.gov.co"www.colciencias.gov.co 2009). Para avanzar en los procesos de aprendizaje significativo que respondan a las necesidades del entorno, se está consolidando, conjunta- mente con el SENA y la Alcaldía de Zarzal, la Tecnoacademia Agrópolis del Norte. La Tecnoacademia es un centro de formación científica donde los jóvenes en edad escolar exploran áreas de alta tecnología en la ingeniería, biotecnología y nanotecnología, involucrado a procesos de innovación e investigación aplicados a la agroindustria. Esta Tecnoacademia sería la primera en el suroccidente colombiano y la segunda en toda Colombia. La Tecnoacademia Cazucá (primera en Colombia) sería un modelo pedagógico replicable en la Agrópolis del Norte. Más información sobre Tecnoacademia Cazucá en: HYPERLINK "http://www.youtube.com/watch ?v=sNTIFCgZCzo\&feature=related"http://www.youtube. $\mathrm{com} /$ watch? $\mathrm{v}=\mathrm{sNTIFCgZCzo \& feature= \text {related }}$ 